\title{
Transmission of methicillin-resistant staphylococcus aureus in the long term care facilities in Hong Kong
}

Vincent CC Cheng ${ }^{1,2}$, Josepha WM Tai ${ }^{2}$, Zoie SY Wong ${ }^{3}$, Jonathan HK Chen ${ }^{1}$, Kris BQ Pan ${ }^{3}$, Yizhen Hai ${ }^{3}$, Wing-Chun $\mathrm{Ng}^{4}$, Denise MK Chow ${ }^{5}$, Miranda CY Yau', Jasper FW Chan ${ }^{1,2}$, Sally CY Wong ${ }^{1,2}$, Herman Tse ${ }^{1,6}$, Sophia SC Chan ${ }^{5}$, Kwok-Leung Tsui ${ }^{3}$, Felix HW Chan ${ }^{4}$, Pak-Leung Ho ${ }^{1,6}$ and Kwok-Yung Yuen ${ }^{1,6^{*}}$

\begin{abstract}
Background: The relative contribution of long term care facilities (LTCFs) and hospitals in the transmission of methicillin-resistant Staphylococcus aureus (MRSA) is unknown.

Methods: Concurrent MRSA screening and spa type analysis was performed in LTCFs and their network hospitals to estimate the rate of MRSA acquisition among residents during their stay in LTCFs and hospitals, by colonization pressure and MRSA transmission calculations.

Results: In 40 LTCFs, 436 (21.6\%) of 2020 residents were identified as 'MRSA-positive'. The incidence of MRSA transmission per 1000-colonization-days among the residents during their stay in LTCFs and hospitals were 309 and 113 respectively, while the colonization pressure in LTCFs and hospitals were 210 and 185 per 1000-patient-days respectively. MRSA spa type t1081 was the most commonly isolated linage in both LTCF residents $(76 / 121,62.8 \%)$ and hospitalized patients (51/87, 58.6\%), while type t4677 was significantly associated with LTCF residents (24/121, $19.8 \%)$ compared with hospitalized patients $(3 / 87,3.4 \%)(p<0.001)$. This suggested continuous transmission of MRSA t4677 among LTCF residents. Also, an inverse linear relationship between MRSA prevalence in LTCFs and the average living area per LTCF resident was observed (Pearson correlation $-0.443, p=0.004$ ), with the odds of patients acquiring MRSA reduced by a factor of 0.90 for each 10 square feet increase in living area.
\end{abstract}

Conclusions: Our data suggest that MRSA transmission was more serious in LTCFs than in hospitals. Infection control should be focused on LTCFs in order to reduce the burden of MRSA carriers in healthcare settings.

\section{Background}

Methicillin-resistant Staphylococcus aureus (MRSA) has emerged worldwide as an important nosocomial pathogen since 1980s [1]. The transfer of colonized or infected patients between hospitals, and repeated hospital admissions were identified to be the two major causes of nosocomial MRSA acquisition [2,3]. Other risk factors for hospital-acquired MRSA include antibiotic exposure, length of hospital stay, admission to intensive care unit (ICU), colonization pressure,

\footnotetext{
* Correspondence: kyyuen@hkucc.hku.hk

'Department of Microbiology, Queen Mary Hospital, Hong Kong Special Administrative Region, China

${ }^{6} \mathrm{Carol}$ Yu Centre for Infection, The University of Hong Kong, Hong Kong

Special Administrative Region, China

Full list of author information is available at the end of the article
}

and underlying co-morbidities. Hence, implementation of antimicrobial stewardship program, hand hygiene campaign, and the use of a bundle approach in the adult ICU were highly recommended for the effective control of nosocomial MRSA transmission [4-7].

In Hong Kong, the increasing number of elderly persons urged the need for long term institutional care and frequent hospitalizations. Long term care facilities (LTCFs) providing skilled nursery services for the elderlies in Hong Kong were found to be a major reservoir for MRSA. The prevalence of MRSA carriers among LTCF residents in Hong Kong was 2.8\% to 5.1\% in 2005 [8,9]. Our recent study showed that $46 \%$ of patients with positive MRSA screening upon hospital admission were LTCF residents [10]. Other studies focusing on the

\section{Biomed Central}


prevalence and risk factors for MRSA colonization have also observed that a recent history of hospitalization is an important determinant for MRSA colonization among the population within LTCFs $[8,11-18]$. However, the relative contribution of LTCFs and hospitals in the degree of MRSA transmission within the healthcare setting is undetermined.

In this study, we analyzed the acquisition of MRSA within LTCFs and hospitals in our locality. The findings of this study may have significant implication for infection control planning and resource allocation in the LTCFs.

\section{Methods}

\section{Study design}

This study compared (i) the prevalence and risk factors of MRSA colonization between the LTCFs and hospitals, and (ii) the incidence of MRSA transmission per 1000colonization-days among the resident during their stay in LTCFs and hospitals. Furthermore, the transmission of MRSA was analyzed by Staphylococcus protein A (spa) typing, and the relationship between MRSA prevalence and living area per LTCF resident was also evaluated in this study.

\section{Setting and participants}

A prospective study was conducted from 1 July to 31 December 2011 to determine the prevalence and acquisition of MRSA among LTCFs and their network hospitals in the Hong Kong West Cluster, which served a population of 0.53 million. LTCFs is a collective term for all long term nursing facilities that provide daily nursing care for their residents including the use of feeding tubes, urinary catheters and other medical devices. The hospital network in our healthcare region included a tertiary referral university-affiliated acute hospital with 1600 beds, 3 extended-care hospitals with a total of 1600 beds, and 1 pediatric hospital. Patients from LTCFs within our healthcare region are admitted to the acute hospital within the region for management. Once stabilized, patients would either be discharged to their original LTCFs or transferred to one of the 3 extended-care hospitals within the regional hospital network before returning to the LTCFs. Community geriatric assessment team, comprising of geriatricians, nurses and allied health professionals, would provide regular on-site visits to the LTCFs within our healthcare region for comprehensive medical follow-up and provide recommendations on infection control measures. In this study, we recruited all residents who agreed to join this study from the 57 LCTFs under the care of the community geriatric assessment team in our healthcare region. Also, we included nasal MRSA screening results of patients admitted to the acute hospital within the study period between 1 July and 31 December 2011 into the study for analysis.
This study protocol has been approved by the Institutional Review Board of the University of Hong Kong/ Hospital Authority Hong Kong West Cluster (IRB reference number: UW 11-235).

\section{Data collection}

The objectives of the study and procedures involved were explained to the community geriatric assessment team and LTCFs representatives in our healthcare region. Informed consent was directly obtained from each of the participating residents, or their relatives if the resident was mentally incapacitated. Student nurses were recruited for specimen collection at the LTCFs and hospital admission wards between 1 July and 31 August 2011. They were trained by infection control nurses on the techniques in taking nasal swabs according to a standard protocol as previously described [19]. Patients' demographic information, history of hospitalization, underlying conditions, and the presence of indwelling devices, wounds or ulcers, were collected from patients' charts and hospital computer information system. Antimicrobial treatment history within the preceding three months of MRSA screening was also analyzed.

To obtain the number of MRSA carriers among the LTCF residents being hospitalized, MRSA screening from nasal swabs were taken within 24 hours when the the LTCF residents admitted to our acute hospital. Acquisition of MRSA in LTCFs was defined as a negative MRSA screening at the LTCFs between 1 July and 31 August 2011 followed by a positive result upon hospital admission screening. The time interval between the first negative sample collection at the LTCFs and the positive hospital admission screening was recorded. Similarly, to investigate the nosocomial MRSA acquisition among LTCF residents during their hospital stay, nasal swabs for MRSA screening were repeated at the acute and 3 extended-care hospitals before being discharged to the patients' respective LTCFs. Nosocomial acquisition of MRSA was defined as the conversion of nasal MRSA carriage status from negative to positive during hospitalization. The time interval between hospital admission and discharge was recorded.

The MRSA colonization pressure in different patient groups is estimated using the formula for calculating colonization pressures per 1000-LTCFs resident-days, as described previously [20]. The colonization pressure for LTCF residents was defined as the ratio of MRSAcarrying LTCF resident-days over the total number of LTCF resident-days, while the colonization pressure for hospitalized LTCF residents was defined as the ratio of imported-MRSA hospitalized-days over the total number of hospitalized days during the study. The incidence of MRSA transmission during their stay in LTCFs and in hospitals were measured in terms of MRSA transmission 
per 1000-colonization-days. The data on the total number of LTCFs resident-days and hospitalized days were collected from the community geriatric assessment team and the hospital record office respectively.

\section{Data analysis}

To determine the differences between patients with probable LTCF-acquired MRSA and probable hospital-acquired MRSA, patients were classified into "LTCFs subgroup" and "hospital subgroup" for further analysis. LTCF residents who had no history of hospitalization in the past 12 months are classified as 'LTCFs subgroup', while "hospital subgroup" consisted of non-LTCF patients who were admitted to the acute hospital within the study period. An exposure window of 12 months was selected as the length of monitoring period, since the median carriage of MRSA was found to be 8.5 months after hospital discharge [21], and the 12-months period has also been adopted in other MRSA transmission epidemiology studies [22,23]. The risk factors for MRSA acquisition in the LTCFs subgroup were analyzed, and the MRSA spa type distribution between LTCFs and hospital subgroups were compared.

As the general demographic factors showed no significant difference on MRSA acquisition between the two subgroups, we sought for other potential LTCFs specific contributing factor. Hong Kong is a highly populated city with limited land resource and LTCFs are of great demand, therefore LTCFs are often crowded. Thus, we postulate that living area may affect the living standard of the elderly and the average living area in LTCFs may correlate with the hygienic standard of the LTCFs in Hong Kong. The overall MRSA prevalence in LTCFs was compared with the average living area (square feet per person) per resident of different LTCFs. The size of each LTCF was estimated from the government registrations and commercial websites for property trading and anonymous on-site assessment was made by two co-authors to validate the information. The official capacity and occupancy of each LTCF was collected from the community geriatric assessment team. The living area per person was defined as the total area of the LTCF divided by the number of residents at the time of study.

\section{Microbiological analysis}

Swab specimens collected from the study subjects were delivered to the laboratory immediately for inoculation on MRSA chromID culture media (bioMérieux), which was incubated aerobically at $35^{\circ} \mathrm{C}$ for 48 hours. MRSA colonies were confirmed as previously described [19]. DNA was extracted from $S$. aureus colonies using alkaline lysis method and spa typing was performed on the first isolate from each person as previously described $[9,10,24]$.
Repeat sequences were analyzed according to the Ribosomal Differentiation of Micro-organisms (RIDOM) database on Staphylococcus aureus (http://www.ridom.de/staphtype) for spa typing.

\section{Statistical analysis}

For statistical calculation, the Chi-square test, Fisher's exact test, $t$-test, or the Mann-Whitney $U$-test was used where appropriate. Pearson correlation was calculated to evaluate the potential linear relationship between overall MRSA prevalence in the LTCFs and the average living area (square feet per person) per LTCF resident. All reported $\mathrm{p}$-values were two-sided. A p-value of $<0.05$ was considered statistically significant. Computation was performed using the Predictive Analytics Soft Ware (PASW) Version 18.0 (formerly SPSS) for Windows and R 2.14.0.

\section{Results}

\section{Prevalence and risk factors for MRSA colonization in LTCFs and hospitals}

Of the 57 LTCFs under the coverage of community geriatric assessment service at our healthcare region, 40 (70.2\%) LTCFs participated in our study. The LTCF residents had to share toilet facilities. Nursing care was provided by on-site staff but the medical problems were taken care of by the community geriatric assessment team who visits at regular basis. Thirteen percent of residents have in situ feeding tubes, urinary catheters or other medical devices requiring special care. During the study period, 2900 residents lived in these LTCFs, of which 2020 residents (69.7\%) consented for the study. Among the 2020 recruited residents, 436 of them (21.6\%) were identified to be MRSA positive (Figure 1) through the LTCFs on-site surveillance screening. Compared with the other 1584 recruited residents without MRSA colonization, MRSA carriers had significantly more episodes of hospitalization $(72.2 \%$ vs $53.7 \%, \mathrm{p}<0.001)$ and longer cumulative length of hospital stay in the past 12 months (Additional file 1: Table S1).

During the concurrent period, admission MRSA screening performed for all subjects in the study cohort within 24 hour of admission to the acute hospital. Among the patients from 1290 consecutive hospital admissions, 204 (15.8\%) were identified as MRSApositive (Figure 1). A significantly higher proportion of MRSA-positive patients were admitted directly from the LTCFs $(57.4 \%)$ comparing to non-LTCF residents $(13.6 \%) \quad[\mathrm{p}<0.001$; odd ratio of $8.52 \quad(6.15-11.82)$ ] (Additional file 2: Table S2).

Eight hundred and fifty-four (42.3\%) of 2020 LTCF residents with no history of hospitalization in the past 12 months (LTCFs subgroup) and 1025 (79.5\%) of 1290 


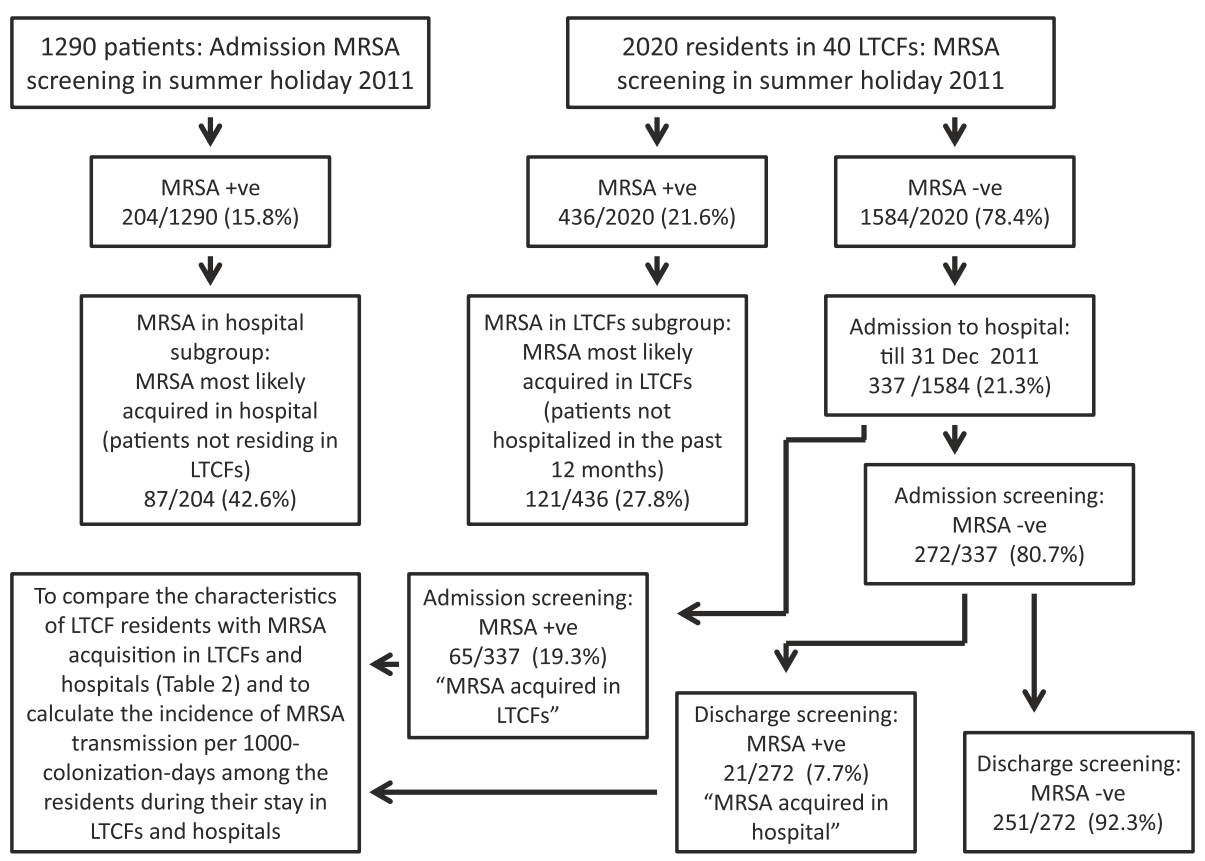

Figure 1 Overview of the MRSA colonization among LTCF residents and hospitalized LTCF residents in the healthcare region, Hong Kong West. Note. LTCFs, Long term care facilities.

non-LTCF hospitalized patients (hospital subgroup) were selected for further analysis to determine the differences between patients with probable LTCF-acquired MRSA and probable hospital-acquired MRSA. From the LTCFs subgroup, $121(14.2 \%)$ of 854 residents were MRSA carriers, while only 87 (8.5\%) of 1025 patients from the hospital subgroup were identified as MRSA carriers (Figure 2). The risk factors for MRSA colonization in the LTCFs and hospital subgroups as determined by logistic regression analysis are shown in Table 1 . Residing in LTCFs was shown to be a significant risk factor for MRSA colonization. Moreover, the presence of urinary catheter, chronic cerebral conditions, the use of $\beta$-lactam $/ \beta$-lactamase inhibitors within three months of MRSA screening were also found to be significant risk factors. After adjusting for the confounding factors, the estimated odds for persons having MRSA in LTCFs were 3.4 times higher than those not residing in LTCFs.

\section{Acquisition of MRSA among residents during their stay in LTCFs and in hospitals}

Among the 1584 LTCF residents who were found to be non-MRSA carriers during the on-site surveillance period between 1 July and 31 August 2011, 337 of them (21.3\%) were subsequently admitted to the acute hospitals, and were subjected to MRSA admission and discharge screening (Figure 1). Admission screening had identified 65/337 (19.3\%) residents to have become MRSA-positive suggesting that they had acquired MRSA in the LTCFs since the time of on-site surveillance. The median time of MRSA detection from surveillance to admission was 77 days (range, 9-181 days). Given that the 436 MRSApositive residents identified during the on-site surveillance had stayed in the 40 LTCFs for 66,802 days, and the overall 2020 residents had stayed in the LTCFs for 317,752 days during our study period, the colonization pressure of MRSA in LTCFs would be 210 per 1000resident-days [(MRSA resident-days of 436 MRSApositive residents was 66,802 days)/(total resident-days of 2020 residents was 317,752 days $) \times 1000$ days]. With the use of these information, the rate of MRSA transmission of the 65 defined LTCFs acquired MRSA was estimated to be 309 MRSA transmissions per 1000-colonization-days among LTCF residents [(65 residents acquired MRSA in LTCFs)/(colonization pressure of 210 per 1000-resident-days) $\times 1000$ days $]$.

During hospitalization, 21 (7.7\%) out of 272 the MRSA-negative LTCF residents acquired MRSA. The median time of MRSA detection was 7 days (ranged 1-31 days). Given that the 65 LTCF-acquired MRSA residents stayed in hospital for a total of 396 days, and the 337 non-MRSA carrying LTCF residents, during the on-site surveillance, stayed for 2137 days during our study period, the MRSA colonization pressure for hospitalized LTCF residents was 185 per 1000-patient-days [(importedMRSA patient-days of 396 days)/(total patient-days of 337 residents of 2137 days) $\times 1000$ days]. Based on this data, we further estimated the rate of MRSA transmission for hospitalized LTCF residents to be 113 MRSA transmissions per 1000-colonization-days [(21 residents 


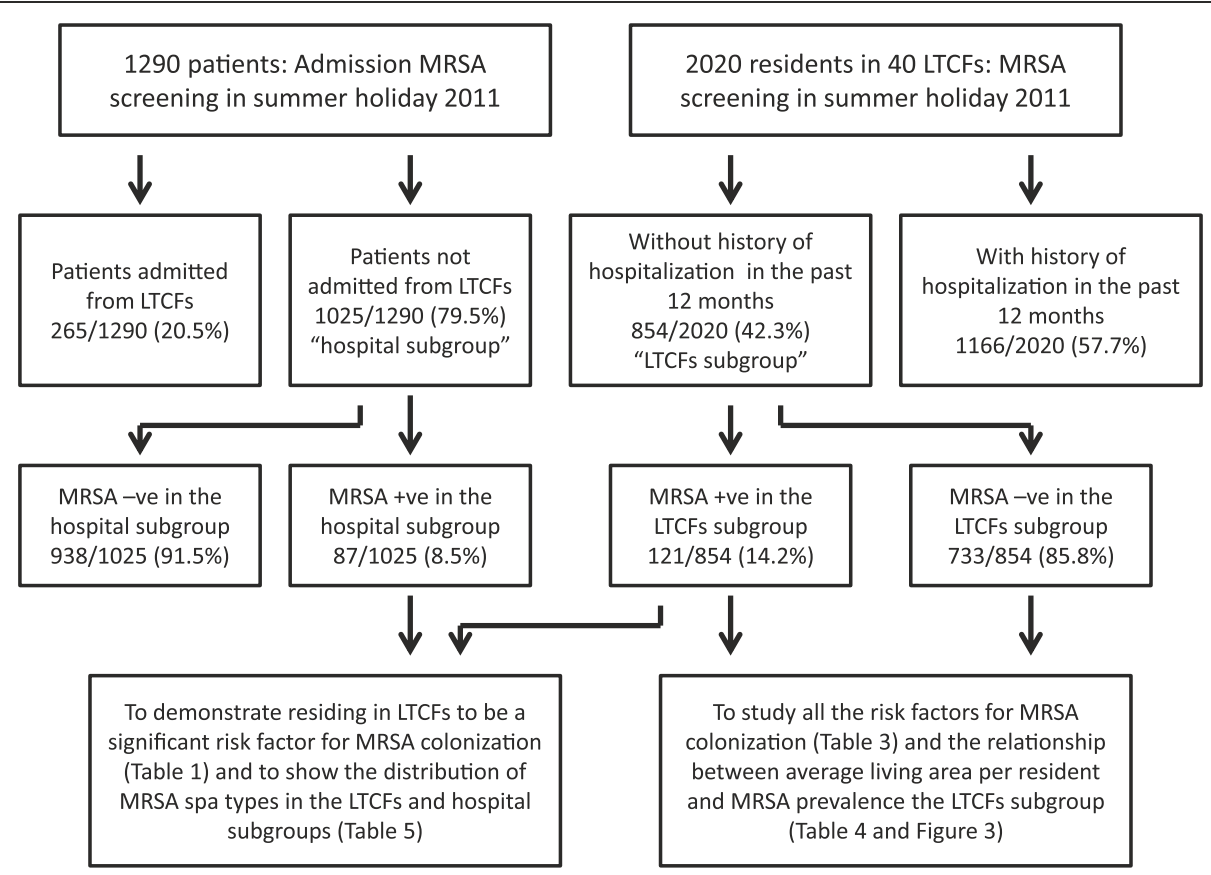

Figure 2 Overview of the logistic of follow up analysis in both LTCF residents and hospitalized patients recruited in our study.

acquired MRSA in hospital)/(colonization pressure of 185 per 1000-patient-days) $\times 1000$ days]. The demographic characteristics of persons with MRSA acquisition in LTCFs and hospitals were not significantly difference (Table 2).

\section{Relationship between MRSA prevalence and living area per LTCFs resident}

An inverse linear relationship between MRSA prevalence in the LTCFs and average living area (square feet per person) per LTCF resident was found (Figure 3). Pearson correlation of MRSA prevalence per LTCF and living area per resident was $-0.443(\mathrm{p}=0.004)$. Risk factors for MRSA colonization in the LTCFs subgroup was shown in Table 3. The odds of patients having MRSA reduced by a factor of 0.90 for each 10 square feet increase in area per person when the other risk factors were held constant (Table 4).

\section{Spa type diversity in LTCFs subgroup and hospital subgroup}

Spa typing was performed for 121 MRSA strains from the LTCFs subgroup and 87 MRSA strains from the hospital subgroup. The spa type diversities were significantly different between the two subgroups (Fisher's exact test, $\mathrm{p}<0.01$ ) (Table 5). The most common spa type was t1081, which constituted 76 (62.8\%) of 121 and 51 (58.6\%) of 87 MRSA strains from the LTCFs and hospital subgroups respectively. Another spa type t4677 was significantly associated with the LTCFs subgroup while t002 was significantly associated with the hospital subgroup. This diversity in spa type was also observed among the

Table 1 Logistic regression analysis for the estimated probability of detection of MRSA with the following risk factors in the combined LTCFs subgroup and hospital subgroup

\begin{tabular}{|c|c|c|c|c|}
\hline & Estimate & Standard error & $z$ value & $p$ value \\
\hline (Intercept) & -3.257 & 0.189 & -17.216 & $<0.001$ \\
\hline Presence of nasogastric tube feeding & 0.643 & 0.286 & 2.251 & 0.024 \\
\hline Presence of urinary catheter & 1.318 & 0.259 & 5.094 & $<0.001$ \\
\hline Chronic cerebral conditions & 0.794 & 0.187 & 4.247 & $<0.001$ \\
\hline Use of $\beta$-lactam/ $\beta$-lactamase inhibitors within 3 months of MRSA screening & 0.860 & 0.224 & 3.843 & $<0.001$ \\
\hline Residence in LTCFs & 1.218 & 0.205 & 5.945 & $<0.001$ \\
\hline
\end{tabular}

LTCFs, long term care facilities.

Hospital subgroup, defined as patients who were not referred from LTCFs; LTCFs subgroup, defined as LTCF residents who had not been hospitalized in the past 12 months.

Note. After adjusting the other confounding factors, the estimated odds for persons having MRSA in LTCFs is exp $(1.218) \approx 3.4$ times than those not residing in LTCFs. 
Table 2 Demographic characteristics of LTCF residents with MRSA acquisition in LTCFs and hospitals

\begin{tabular}{|c|c|c|c|}
\hline & $\begin{array}{l}\text { MRSA acquisition in LTCFs } \\
(n=65)\end{array}$ & $\begin{array}{l}\text { MRSA acquisition in hospitals } \\
(n=21)\end{array}$ & $p$ value \\
\hline Age (mean $\pm S D)$ & $85.1 \pm 10.2$ & $84.2 \pm 10.8$ & 0.744 \\
\hline Sex (male) & $24(36.9 \%)$ & $8(38.1 \%)$ & 0.923 \\
\hline \multicolumn{4}{|l|}{ Underlying diseases } \\
\hline Chronic cerebral conditions $^{a}$ & $29(44.6 \%)$ & 7 (33.3\%) & 0.362 \\
\hline Chronic cardiac conditions $^{b}$ & $8(12.3 \%)$ & $6(28.6 \%)$ & 0.079 \\
\hline Chronic pulmonary conditions $^{c}$ & 7 (10.8\%) & $3(14.3 \%)$ & 0.662 \\
\hline Chronic renal failure & $2(3.1 \%)$ & $2(9.5 \%)$ & 0.223 \\
\hline Liver cirrhosis & 0 & 0 & NA \\
\hline Diabetes mellitus & $11(16.9 \%)$ & $5(23.8 \%)$ & 0.481 \\
\hline Malignancy & $4(6.2 \%)$ & $1(4.8 \%)$ & 0.813 \\
\hline \multicolumn{4}{|l|}{ Presence of } \\
\hline Nasogastric tube & $23(35.4 \%)$ & 7 (33.3\%) & 0.864 \\
\hline Urinary catheter & $15(23.1 \%)$ & $3(14.3 \%)$ & 0.389 \\
\hline Tenckhoff catheter & 0 & 0 & NA \\
\hline Wound or ulcer & $1(1.5 \%)$ & $2(9.5 \%)$ & 0.083 \\
\hline \multicolumn{4}{|c|}{ Antibiotics therapy within 3 months of MRSA screening } \\
\hline Penicillin group & $11(16.9 \%)$ & $6(28.6 \%)$ & 0.244 \\
\hline$\beta$-lactam/ $\beta$-lactamase inhibitors & $23(35.4 \%)$ & $8(38.1 \%)$ & 0.822 \\
\hline Cephalosporin group & $7(10.8 \%)$ & $3(14.3 \%)$ & 0.662 \\
\hline Carbapenem group & 0 & $1(4.8 \%)$ & 0.077 \\
\hline Fluoroquinolones & $2(3.1 \%)$ & $2(9.5 \%)$ & 0.223 \\
\hline
\end{tabular}

LTCFs, residential care homes for elderly; MRSA, methicillin-resistant Staphylococcus aureus; NA, not applicable; ${ }^{\text {a }}$ chronic cerebral conditions included cerebrovascular accident, dementia, and Parkinson disease; ${ }^{\mathrm{b}}$ chronic cardiac conditions included ischemic heart disease and congestive heart failure; c chronic pulmonary conditions included chronic bronchitis, chronic obstructive pulmonary disease, and asthma.

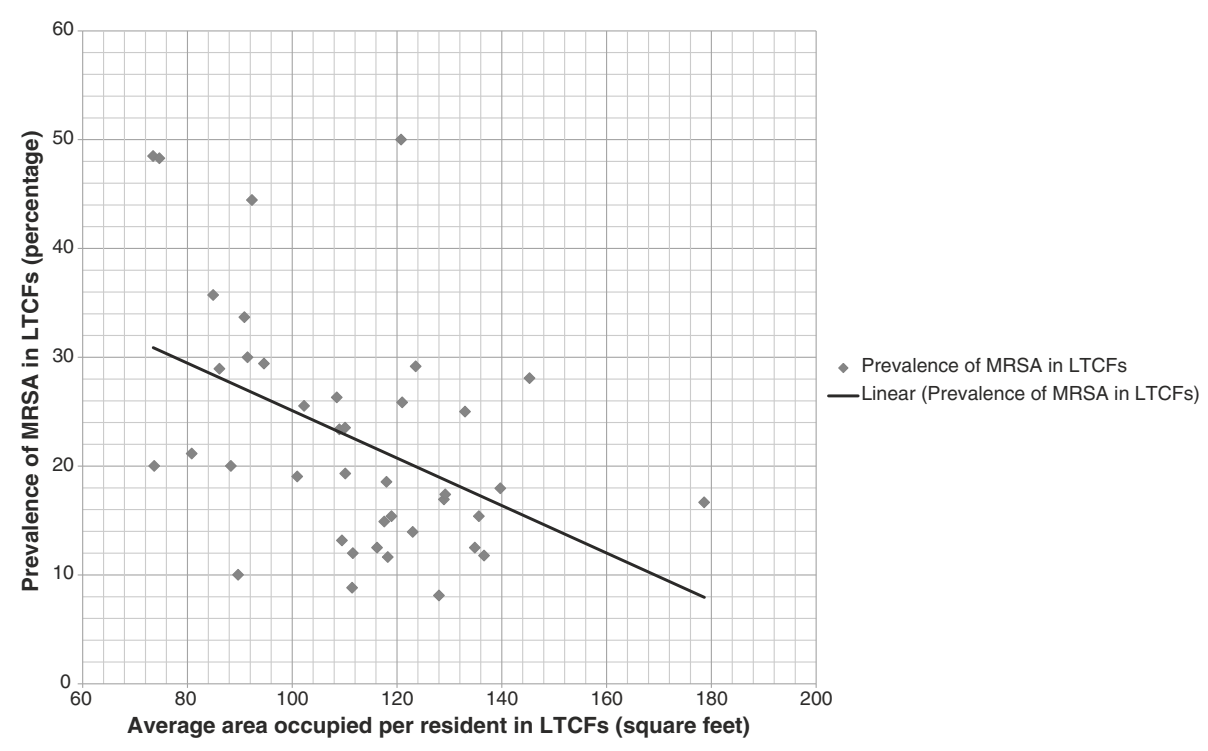

Figure 3 The relationship between the MRSA prevalence per LTCF and the average living area (square feet per person) per LTCF resident. Note. Pearson correlation of MRSA prevalence per LTCFs and area (square feet) per person $=-0.443(p=0.004)$. 
Table 3 Risk factors for MRSA colonization in the LTCFs subgroup

\begin{tabular}{|c|c|c|c|}
\hline & MRSA carrier $(n=121)$ & Non-MRSA carrier $(n=733)$ & $\mathrm{p}$ value \\
\hline Age (mean $\pm S D)$ & $84.3 \pm 8.9$ & $83.0 \pm 10.1$ & 0.144 \\
\hline Sex (male) & $35(28.9 \%)$ & $246(33.6 \%)$ & 0.368 \\
\hline \multicolumn{4}{|l|}{ Underlying diseases } \\
\hline Chronic cerebral conditions $^{a}$ & $25(20.7 \%)$ & $84(11.5 \%)$ & 0.008 \\
\hline \multicolumn{4}{|l|}{ Presence of } \\
\hline Nasogastric tube & $13(10.7 \%)$ & $39(5.3 \%)$ & 0.035 \\
\hline Urinary catheter & $5(4.1 \%)$ & $5(0.7 \%)$ & 0.005 \\
\hline \multicolumn{4}{|c|}{ Antibiotics therapy within 3 months of MRSA screening } \\
\hline$\beta$-lactam/ $\beta$-lactamase inhibitors & $6(5.0 \%)$ & $15(2.0 \%)$ & 0.110 \\
\hline Area (square feet) per person ${ }^{b}$ & $108.8 \pm 20.1$ & $113.0 \pm 19.0$ & 0.031 \\
\hline
\end{tabular}

337 patients who were MRSA-negative during LTCFs on-site surveillance who were subsequently hospitalized within the study period. The 65 patients $(65 / 337,19.3 \%)$ who were identified to be positive with MRSA at the admission screening had spa types which belonged to the LTCFs subgroup, while the 272 (272/337, 80.7\%) who were MRSA-negative at admission screening but later became MRSA-positive at discharge screening had spa types which belonged to the hospital subgroup.

\section{Discussion}

Our study showed that the prevalence of MRSA among LTCFs in Hong Kong had increased substantially from $3-5 \%$ to over 20\% (436 MRSA positive/2020 residents in 40 LTCFs) (Figure 1) in the recent six years $[8,9]$. This finding is comparable to those in the United States [25] study but higher than other studies conducted in Germany [11,18], Belgium [12,16], and Spain [15]. Similar to the previous studies, history of hospitalization, chronic

Table 4 Logistic regression analysis for the estimated probability of detection of MRSA with the following risk factors in the LTCFs subgroup

\begin{tabular}{lllll}
\hline & Estimate & $\begin{array}{l}\text { Standard } \\
\text { error }\end{array}$ & z value & p value \\
\hline (Intercept) & -0.422 & 0.578 & -0.729 & 0.46588 \\
Presence of urinary catheter & 2.125 & 0.660 & 3.219 & 0.00129 \\
Chronic cerebral conditions $^{*}$ & 0.742 & 0.256 & 2.899 & 0.00374 \\
Area (square feet) per person $^{\text {a }}$ & -0.014 & 0.006 & -2.648 & 0.0081 \\
\hline
\end{tabular}

LTCFs, long term care facilities; LTCFs subgroup, defined as LTCF residents who had not been hospitalized in the past 12 months; ${ }^{a}$ area per person is defined as total area of the LTCFs over the number of resident occupying at the time of study.

Note. The odds of a patient having MRSA decreased by exp $(-0.14) \approx 0.90$ times with each 10 square feet increase in area (square feet) per person when the other risk factors of presence of urinary catheter and chronic cerebral conditions were held constant. comorbidity, indwelling devices, wound or ulcer, and antimicrobial therapy were found to be risk factors for MRSA colonization in our study $[11,12,15,16,18]$. Moreover, residence in LTCFs and a long cumulative length of hospital stay in the past 12 months were again found to be significant risk factors for MRSA colonization by univariate analysis in our concurrent admission screening [26-30]. Transfer of patients between LTCFs and hospitals creates a vicious cycle which perpetuates MRSA transmission. Hence, it is of great importance to investigate the relative contribution of LTCFs and hospitals in the transmission dynamics of MRSA in the healthcare setting.

Through this study, we identified that acquisition of MRSA among LTCF residents was 3.4 times higher than those patients who did not reside in LTCFs by multivariate

Table 5 Comparison of MRSA spa type in the LTCFs subgroup and hospital subgroup

\begin{tabular}{lllll}
\hline Spa type & $\begin{array}{l}\text { MRSA in LTCFs } \\
\text { subgroup }\end{array}$ & $\begin{array}{l}\text { MRSA in hospital } \\
\text { subgroup }\end{array}$ & p value & Odd ratio \\
\hline t002 & $2(1.7 \%)$ & $14(16.1 \%)$ & $<0.001$ & 0.09 \\
t032 & $2(1.7 \%)$ & $5(5.7 \%)$ & 0.132 & 0.28 \\
t037 & $2(1.7 \%)$ & $6(6.9 \%)$ & 0.070 & 0.23 \\
t701 & $8(6.6 \%)$ & $1(1.1 \%)$ & 0.083 & 6.05 \\
t1081 & $76(62.8 \%)$ & $51(58.6 \%)$ & 0.567 & 1.19 \\
t4677 & $24(19.8 \%)$ & $3(3.4 \%)$ & $<0.001$ & 6.88 \\
Other & $7(5.8 \%)^{a}$ & $7(8.0 \%)$ & NA & NA \\
Total & $121(100 \%)$ & $87(100 \%)$ & NA & NA \\
\hline
\end{tabular}

Hospital subgroup, defined as hospitalized patients who were not referred from LTCFs; NA, not applicable; LTCFs, long term care facilities; LTCFs subgroup, defined as LTCF residents who had not been hospitalized in the past 12 months; ${ }^{a}$ including spa types of t121 (2), t012 (1), t1026 (1), t1765 (1), t2536 (1), and t588 (1); ${ }^{b}$ including spa types of t437 (2), t1250 (1), t1751 (1), t441 (1), t5413 (1), and t9377 (1). 
analysis. The incidence of MRSA transmission per 1000colonization-days among LTCF residents was also three times higher than that of the hospitalized LTCF residents, given that the colonization pressure in both LTCFs and hospital were similar. Our findings suggested that MRSA transmission in LTCFs was more severe than that in the hospitals.

While there was no difference in risk factors between patients with LTCFs-acquired and hospital-acquired MRSA, it was noted that the average living area per resident in different LTCFs was an important surrogate marker reflecting the hygienic standard of LTCFs. An inverse linear relationship between MRSA prevalence in the LTCFs and the average living area per resident was found. Provided that the other risk factors were held constant, the odds of patients acquiring MRSA is reduced by a factor of 0.9 for each increment of 10 square feet in living area. To our best knowledge, our study is the first quantitative analysis to demonstrate that living area per person could be a determinant of MRSA prevalence in LTCFs. This finding is particularly relevant for urban cities with a high population density like Hong Kong with an average land price of USD 1000 per square feet. The supply of residential land is limited and living environments are characterized by extremely compact multi-storey apartments [31]. The average living area is an important indicator of the degree of spatial separation, making it a suitable surrogate marker of LTCFs in terms of the overall standard of care, hygiene and infection control. Indeed, overcrowded environment in the correctional facility had been implicated as a contributing factor for MRSA transmission in the United States [32,33].

The spa typing results of MRSA strains collected from the LTCFs and hospital subgroups showed that two distinct spa type linages, t4677 and t002, were significantly associated with the LTCFs subgroup and the hospital subgroup respectively. Type t002 was commonly found in hospitalized patients in our region and the United States [10,34,35] whereas type $t 4677$ had not been reported in the community or hospital setting previously. This might suggest that 44677 isolates circulated exclusively among LTCF residents in our locality. On the other hand, the high predominance of type t1081 (about $60 \%$ of isolates) in both the LTCFs and hospital subgroups could be explained by the intrinsically high transmissibility of t1081 as shown in our previous study [10]. Alternatively, t1081 might have been introduced into our healthcare system in the early years allowing cross-transmission among LTCF residents and hospitalized patients. In fact, t1081 had been predominantly found in our LTCFs in 2005 [9], and t1081, as a member of ST45/Staphylococcus cassette chromosome mec (SCCmec) IV or V, was increasingly reported in our hospital isolates from 1995 to 2005 [36].
There are several limitations in our study. Firstly, only $70 \%$ of the LTCF residents consented for this study introducing potential bias in subject selection. In addition, we only collected nasal specimens for MRSA screening due to resource limitation. Furthermore, the sensitivity of detection [37] may be compromised in subjects with low microbial load while not on antibiotic therapy $[19,38]$. Chromogenic agar, however, was used to improve sensitivity and cost-effectiveness [39]. We did not analyze the staffing ratio in LTCFs, which might affect the prevalence of MRSA [40]. As the LTCFs have to satisfy the basic infection control measures required by the government, living area per resident was chosen as an important surrogate marker reflecting the hygienic standard of LTCFs. We did not screen for MRSA carriage among healthcare workers in the LTCFs and hospitals since the benefit of carriage eradication is not established in nonoutbreak setting [41], despite a recent study suggesting that both residents and staff were involved in MRSA transmissions [42]. In addition, this is a single season study and may not be applicable to other seasons.

\section{Conclusion}

In summary, we had established the relative importance of LTCFs in the transmission dynamics of MRSA between LTCFs and hospitals in the healthcare setting. More resources should be allocated to improve the infection control measures of LTCFs and further studies are necessary to understand key factors, such as space availability, that lead to high level of MRSA transmission within LTCFs.

\section{Additional files}

Additional file 1: Table S1. Demographic characteristic of 2020 residents from the 40 LTCFs in the Hong Kong West region. Note. LTCFs, long term care facilities; SD, standard deviation.

Additional file 2: Table S2. Demographic characteristic of 1290 consecutive patients (from both LTCFs and non-LTCFs) with or without MRSA colonization upon admission. Note. LTCFs, long term care facilities; $\mathrm{SD}$, standard deviation.

\section{Competing interests}

The authors declare that they have no competing interests.

\section{Authors' contributions}

VCCC and KYY designed, executed and supervised the study. VCCC, JWMT, WCN, DMKC, SSCC, and FHWC coordinated collection of clinical specimens in the long term care facilities. VCCC, JWMT, and JFWC coordinated collection of clinical specimens upon hospital admission. JHKC and MCYY conducted molecular characterization of MRSA strains and validated the area of long term care facilities. ZSYW, KBQP, and HT performed the statistical analysis. VCCC drafted the manuscript. JFWC, SCYW, HT, KLT, PLH and KYY critically reviewed the manuscript. All authors have read and approved the final manuscript

\section{Acknowledgment}

We thank JKH Luk, PKC Chiu, CKY Ho, TC Law, WK Chan, WY Chan, J Chiu, SM Leung, SM Woo of Community Care Service Team, and WK Lo, PC Wong 
of Infection control team, in facilitating this study. The work was partially supported by the Research Fund for the Control of Infectious Diseases of the Food and Health Bureau of the Hong Kong SAR Government.

\section{Author details}

'Department of Microbiology, Queen Mary Hospital, Hong Kong Special Administrative Region, China. ${ }^{2}$ Infection Control Team, Queen Mary Hospital, Hong Kong Special Administrative Region, China. ${ }^{3}$ Department of Systems Engineering and Engineering Management, City University of Hong Kong, Hong Kong Special Administrative Region, China. ${ }^{4}$ Community Geriatric Assessment Team, Fung Yiu King Hospital, Hong Kong Special Administrative Region, China. ${ }^{5}$ School of Nursing, The University of Hong Kong, Hong Kong Special Administrative Region, China. ${ }^{6} \mathrm{Carol}$ Yu Centre for Infection, The University of Hong Kong, Hong Kong Special Administrative Region, China.

Received: 15 June 2012 Accepted: 2 May 2013

Published: 6 May 2013

\section{References}

1. Ayliffe GA: The progressive intercontinental spread of methicillinresistant Staphylococcus aureus. Clin Infect Dis 1997, 24(Suppl 1):S74-79.

2. Thompson RL, Cabezudo I, Wenzel RP: Epidemiology of nosocomial infections caused by methicillin-resistant Staphylococcus aureus. Ann Intern Med 1982, 97(3):309-317.

3. Bradley SF, Terpenning MS, Ramsey MA, Zarins LT, Jorgensen KA, Sottile WS, Schaberg DR, Kauffman CA: Methicillin-resistant Staphylococcus aureus: colonization and infection in a long-term care facility. Ann Intern Med 1991, 115(6):417-422.

4. Cheng VC, To KK, Li IW, Tang BS, Chan JF, Kwan S, Mak R, Tai J, Ching P, Ho $P L$, et al: Antimicrobial stewardship program directed at broad-spectrum intravenous antibiotics prescription in a tertiary hospital. Eur J Clin Microbiol Infect Dis 2009, 28(12):1447-1456.

5. Cheng VC, Tai JW, Ho SK, Chan JF, Hung KN, Ho PL, Yuen KY: Introduction of an electronic monitoring system for monitoring compliance with Moments 1 and 4 of the WHO "My 5 Moments for Hand Hygiene" methodology. BMC Infect Dis 2011, 11:151

6. Tai JW, Mok ES, Ching PT, Seto WH, Pittet D: Nurses and physicians' perceptions of the importance and impact of healthcare-associated infections and hand hygiene: a multi-center exploratory study in Hong Kong. Infection 2009, 37(4):320-333.

7. Cheng VC, Tai JW, Chan WM, Lau EH, Chan JF, To KK, Li IW, Ho PL, Yuen KY: Sequential introduction of single room isolation and hand hygiene campaign in the control of methicillin-resistant Staphylococcus aureus in intensive care unit. BMC Infect Dis 2010, 10:263.

8. Ho PL, Wang TK, Ching P, Mak GC, Lai E, Yam WC, Seto WH: Epidemiology and genetic diversity of methicillin-resistant Staphylococcus aureus strains in residential care homes for elderly persons in Hong Kong. Infect Control Hosp Epidemiol 2007, 28(6):671-678.

9. Ho PL, Lai EL, Chow KH, Chow LS, Yuen KY, Yung RW: Molecular epidemiology of methicillin-resistant Staphylococcus aureus in residential care homes for the elderly in Hong Kong. Diagn Microbiol Infect Dis 2008, 61(2):135-142.

10. Cheng VC, Chan JF, Lau EH, Yam WC, Ho SK, Yau MC, Tse EY, Wong AC, Tai JW, Fan ST, et al: Studying the transmission dynamics of meticillinresistant Staphylococcus aureus in Hong Kong using spa typing. J Hosp Infect 2011, 79(3):206-210.

11. von Baum H, Schmidt C, Svoboda D, Bock-Hensley O, Wendt C: Risk factors for methicillin-resistant Staphylococcus aureus carriage in residents of German nursing homes. Infect Control Hosp Epidemiol 2002, 23(9):511-515.

12. Suetens C, Niclaes L, Jans B, Verhaegen J, Schuermans A, Van Eldere J, Vandenbroucke JP, Buntinx F: Determinants of methicillin-resistant Staphylococcus aureus carriage in nursing homes. Age Ageing 2007, 36(3):327-330

13. Barr B, Wilcox MH, Brady A, Parnell P, Darby B, Tompkins D: Prevalence of methicillin-resistant Staphylococcus aureus colonization among older residents of care homes in the United Kingdom. Infect Control Hosp Epidemiol 2007, 28(7):853-859.

14. Brugnaro P, Fedeli U, Pellizzer G, Buonfrate D, Rassu M, Boldrin C, Parisi SG Grossato A, Palu G, Spolaore P: Clustering and risk factors of methicillinresistant Staphylococcus aureus carriage in two Italian long-term care facilities. Infection 2009, 37(3):216-221.
15. Mariscal D, Dominguez MA, Perez $J$ L, Segura F, Pujol M, Ruiz De Gopegui E, Manzur A, Gavalda L: Prevalence of methicillin-resistant Staphylococcus aureus and factors associated with colonization among residents in community long-term-care facilities in Spain. Clin Microbiol Infect 2008, 14(9):867-872.

16. Denis O, Jans B, Deplano A, Nonhoff C, De Ryck R, Suetens C, Struelens MJ: Epidemiology of methicillin-resistant Staphylococcus aureus (MRSA) among residents of nursing homes in Belgium. J Antimicrob Chemother 2009, 64(6):1299-1306.

17. Lasseter G, Charlett A, Lewis D, Donald I, Howell-Jones R, McNulty CA: Staphylococcus aureus carriage in care homes: identification of risk factors, including the role of dementia. Epidemiol Infect 2010, 138(5):686-696.

18. Pfingsten-Wurzburg S, Pieper DH, Bautsch W, Probst-Kepper M: Prevalence and molecular epidemiology of meticillin-resistant Staphylococcus aureus in nursing home residents in northern Germany. J Hosp Infect 2011, 78(2):108-112.

19. Cheng VC, Li IW, Wu AK, Tang BS, Ng KH, To KK, Tse H, Que TL, Ho PL, Yuen KY: Effect of antibiotics on the bacterial load of meticillin-resistant Staphylococcus aureus colonisation in anterior nares. J Hosp Infect 2008, 70(1):27-34.

20. Eveillard M, Lancien E, Hidri N, Barnaud G, Gaba S, Benlolo JA, Joly-Guillou ML: Estimation of methicillin-resistant Staphylococcus aureus transmission by considering colonization pressure at the time of hospital admission. J Hosp Infect 2005, 60(1):27-31.

21. Scanvic A, Denic L, Gaillon S, Giry P, Andremont A, Lucet JC: Duration of colonization by methicillin-resistant Staphylococcus aureus after hospital discharge and risk factors for prolonged carriage. Clin Infect Dis 2001, 32(10):1393-1398

22. Harbarth S, Sax H, Fankhauser-Rodriguez C, Schrenzel J, Agostinho A, Pittet D: Evaluating the probability of previously unknown carriage of MRSA at hospital admission. Am J Med 2006, 275(3):e215-223. 119.

23. Furuno JP, McGregor JC, Harris AD, Johnson JA, Johnson JK, Langenberg P, Venezia RA, Finkelstein J, Smith DL, Strauss SM, et al: Identifying groups at high risk for carriage of antibiotic-resistant bacteria. Arch Intern Med 2006, 166(5):580-585.

24. Ho PL, Chuang SK, Choi YF, Lee RA, Lit AC, Ng TK, Que TL, Shek KC, Tong HK, Tse CW, et al: Community-associated methicillin-resistant and methicillin-sensitive Staphylococcus aureus: skin and soft tissue infections in Hong Kong. Diagn Microbiol Infect Dis 2008, 61(3):245-250.

25. Reynolds C, Quan V, Kim D, Peterson E, Dunn J, Whealon M, Terpstra L,

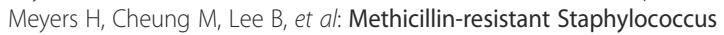
aureus (MRSA) carriage in 10 nursing homes in Orange County California. Infect Control Hosp Epidemiol 2011, 32(1):91-93.

26. Samad A, Banerjee D, Carbarns N, Ghosh S: Prevalence of methicillinresistant Staphylococcus aureus colonization in surgical patients, on admission to a Welsh hospital. J Hosp Infect 2002, 51(1):43-46.

27. Gopal Rao G, Michalczyk P, Nayeem N, Walker G, Wigmore L: Prevalence and risk factors for meticillin-resistant Staphylococcus aureus in adult emergency admissions-a case for screening all patients? J Hosp Infect 2007, 66(1):15-21.

28. Hidron Al, Kourbatova EV, Halvosa JS, Terrell BJ, McDougal LK, Tenover FC, Blumberg HM, King MD: Risk factors for colonization with methicillinresistant Staphylococcus aureus (MRSA) in patients admitted to an urban hospital: emergence of community-associated MRSA nasal carriage. Clin Infect Dis 2005, 41(2):159-166.

29. Haley CC, Mittal D, Laviolette A, Jannapureddy S, Parvez N, Haley RW: Methicillin-resistant Staphylococcus aureus infection or colonization present at hospital admission: multivariable risk factor screening to increase efficiency of surveillance culturing. J Clin Microbiol 2007, 45(9):3031-3038.

30. Eveillard M, Ernst C, Cuviller S, Lescure FX, Malpaux M, Defouilloy Gresanleux M, Duboisset M, Lienard J, Eb F: Prevalence of methicillinresistant Staphylococcus aureus carriage at the time of admission in two acute geriatric wards. J Hosp Infect 2002, 50(2):122-126.

31. Chan EY, Kim JH, Griffiths SM, Lau JT, Yu I: Does living density matter for nonfatal unintentional home injury in Asian urban settings? Evidence from Hong Kong. J Urban Health 2009, 86(6):872-886.

32. Baillargeon J, Kelley MF, Leach CT, Baillargeon G, Pollock BH: Methicillinresistant Staphylococcus aureus infection in the Texas prison system. Clin Infect Dis 2004, 38(9):e92-95.

33. Pan ES, Diep BA, Carleton HA, Charlebois ED, Sensabaugh GF, Haller BL, Perdreau-Remington F: Increasing prevalence of methicillin-resistant Staphylococcus aureus infection in California jails. Clin Infect Dis 2003, 37(10):1384-1388. 
34. Tenover FC, Tickler IA, Goering RV, Kreiswirth BN, Mediavilla JR, Persing DH: Characterization of nares and blood culture isolates of methicillinresistant staphylococcus aureus from patients in United States hospitals. Antimicrob Agents Chemother 2011, 56(3):1324-1330.

35. Ho CM, Ho MW, Lee CY, Tien N, Lu JJ: Clonal spreading of methicillinresistant SCCmec Staphylococcus aureus with specific spa and dru types in central Taiwan. Eur J Clin Microbiol Infect Dis 2012, 31(4):499-504.

36. Ho PL, Chow KH, Lo PY, Lee KF, Lai EL: Changes in the epidemiology of methicillin-resistant Staphylococcus aureus associated with spread of the ST45 lineage in Hong Kong. Diagn Microbiol Infect Dis 2009, 64(2):131-137.

37. Sewell DL, Potter SA, Jacobson CM, Strausbaugh $\sqcup$, Ward TT: Sensitivity of surveillance cultures for the detection of methicillin-resistant Staphylococcus aureus in a nursing-home-care unit. Diagn Microbiol Infect Dis 1993, 17(1):53-56.

38. Cheng VC, Chan JF, To KK, Tai JW, Ho PL: Detection of community-associated MRSA as a result of the unmasking effect of antibiotic treatment. J Hosp Infect 2009, 72(3):273-274.

39. Wassenberg MW, Kluytmans JA, Box AT, Bosboom RW, Buiting AG, van Elzakker EP, Melchers WJ, van Rijen MM, Thijsen SF, Troelstra A, et al: Rapid screening of methicillin-resistant Staphylococcus aureus using PCR and chromogenic agar: a prospective study to evaluate costs and effects. Clin Microbiol Infect 2010, 16(12):1754-1761.

40. Loeb MB, Craven S, McGeer AJ, Simor AE, Bradley SF, Low DE, ArmstrongEvans M, Moss LA, Walter SD: Risk factors for resistance to antimicrobial agents among nursing home residents. Am J Epidemiol 2003, 157(1):40-47.

41. Hawkins G, Stewart S, Blatchford O, Reilly J: Should healthcare workers be screened routinely for meticillin-resistant Staphylococcus aureus? A review of the evidence. J Hosp Infect 2011, 77(4):285-289.

42. Schwaber MJ, Masarwa S, Navon-Venezia S, Kandlik Y, Chmelnitsky I, Smollan G, Glick R, Neria G, Carmeli Y: High prevalence of methicillin-resistant Staphylococcus aureus among residents and staff of long-term care facilities, involving joint and parallel evolution. Clin Infect Dis 2011 , 53(9):910-913.

doi:10.1186/1471-2334-13-205

Cite this article as: Cheng et al:: Transmission of methicillin-resistant staphylococcus aureus in the long term care facilities in Hong Kong. BMC Infectious Diseases 2013 13:205.

\section{Submit your next manuscript to BioMed Central and take full advantage of:}

- Convenient online submission

- Thorough peer review

- No space constraints or color figure charges

- Immediate publication on acceptance

- Inclusion in PubMed, CAS, Scopus and Google Scholar

- Research which is freely available for redistribution 